\title{
Applying the Modified Perturbation Theory to High Energy Scattering in the Quasi-Potential Approach
}

\author{
Nguyen Suan Han $^{1}$, Nguyen NhuXuan ${ }^{1,2}$, and Vu ToanThang ${ }^{1}$ \\ 1. Department of Theoretical Physics, Hanoi National University,344 Nguyen Trai Street, 1000 Hanoi, Vietnam \\ 2. Department of Physics, Le Qui Don University, 236 Hoang Quoc Viet Street, 10000 Hanoi, Vietnam
}

\begin{abstract}
Asymptotic behavior of the scattering amplitude for two scalar particles at high energy and fixed momentum transfer is reconsidered in quantum field theory. In the framework of the quasi-potential approach and the modified perturbation theory, a systematic scheme for finding the leading eikonal scattering amplitudes and its corrections are developed and constructed. The differential cross section is also discussed.
\end{abstract}

Key words: Scattering, quasi-potential, eikonal.

\section{Introduction}

In quantum field theory, interaction of two relativistic particles is investigated using the Bethe-Salpeter covariant equation [1]. It can be found in many problems studying the structure of linking states in crystal or finding the high energy scattering amplitude [2, 3]. However, this approach has several difficulties:

(1) The Bethe-Salpeter wave function has two time variables. Therefore it is difficult to explain in quantum mechanics where scattering amplitude is the transition probability of a quantum system from one state to another. For this reason, the problem of re-normalization is not trivial. So far a complete understanding of this characteristic has not yet been achieved $[4,5]$.

(2) Application of this equation to actual physical processes using ladder approximation does not produce the same results as those using the relativistic equations in quantum mechanics (Klein-Gordon or Dirac) when the mass of one of two particles is very large (approaching infinity) [6].

Corresponding author: Nguyen Nhu Xuan, Ph.D., research field: high energy scattering.
To overcome these difficulties, Logunov and Tavkhelidze have developed a method that relies on the quasi-potential approach to obtain wave function by generalizing the 3D non-relativistic wave function as a function of time satisfying the Schrodinger equation. This method can help us to explore more specific applications that can be solved in more detail $[7,8,9]$ and to develop new approximate methods in the quantum field theory $[4,5,10-12]$.

Obtaining an exact solution for the quasi-potential equation is very difficult. In this paper we develop the modified perturbation theory proposed by Fradkin [12] in quantum field theory for the Logunov-Tavkhelidze equation to find the scattering amplitude of two scalar particles at high energies and fixed momentum transfer.

This paper is organized as follows. In the second section, we briefly introduce the modified perturbation method to solve the quasi-potential equation $[13,14]$. The Logunov-Tavkhelidze equation on a mass shell is rewritten as an operator, after defining the scalar operator. In the third section, the solution of this equation is expressed exponentially which is convenient for using the modified perturbation method. The asymptotic equation of amplitude scattering at high energies and fixed momentum 
transfer is also considered in this section. Section 4 is devoted to computing the scattering amplitude and the differential cross section for specific potentials such as the Yukawa potential and the Gaussian potential. Note that, the collision parameters selected here are less than the wavelength of the scattering particle. Finally, we discuss the results obtained and the generalizability of the method to gravitational interaction.

\section{Quasi Potential Equation in an Operator Form}

The Logunov-Tavkhelidze quasi-potential equation is the basis of quantum field theory. In the 4-dimensional space-time formalism, it was generalized from the Schrodinger equation by using the 2-times Green function.

We examine the elastic scattering of interaction of two scalar nucleons by exchanging scalar mesons. In order to find the scattering amplitude, the Logunov-Tavkhelidze equation with local quasi-potential is written in the form [12-15]:

$$
\begin{array}{r}
T\left(\vec{p}, \vec{p}^{\prime} ; s\right)=g V\left(\vec{p}-\vec{p}^{\prime} ; s\right)+ \\
g \int d \vec{q} K\left(\vec{q}^{2} ; s\right) V(\vec{p}-\vec{q} ; s) T\left(\vec{q}, \vec{p}^{\prime} ; s\right)
\end{array}
$$

where $K\left(\vec{q}^{2} ; s\right)$ is the kernel, $s=4\left(p^{2}+m^{2}\right)=4\left(p^{\prime 2}+m^{2}\right)$ is the total energy and $p, p^{\prime}$ are the relativistic momentum of two particles in the center of mass reference frame in the initial and final states respectively. In general, the quasi-potential is a complex function of the energy and relativistic momentum of two particles. The standard equation becomes simpler if the quasi-potential is "smooth" or otherwise the quasi-potential is a function of the difference between the relativistic momentum two particles and the total energy (called the local quasi-potential) [4]. Existence of the local quasi-potential has been well proven in the case of weak interactions and this is also the way we build it. The local quasi-potential built in this way will yield the solution of Eq. (2.1), which is considered as the physical amplitude of the two-particle scattering on the mass shell.

Performing the Fourier transformation

$$
\begin{aligned}
& V\left(\vec{p}-\vec{p}^{\prime} ; s\right)=\frac{1}{(2 \pi)^{3}} \int d r e^{i(\vec{p}-\vec{p}) r} V(r ; s), \\
& T\left(\vec{p}, \vec{p}^{\prime} ; s\right)=\int d \vec{r} d \vec{r}^{\prime} \exp (i \vec{p} \vec{r}) \exp \left(-i \vec{p}^{\prime} \vec{r}^{\prime}\right) T\left(\vec{r}, \vec{r}^{\prime} ; s\right)
\end{aligned}
$$

and substitute Eq. (2.2) into Eq.(2.1), we have:

$$
T\left(\vec{r}, \vec{r}^{\prime} ; s\right)=\frac{g}{(2 \pi)^{3}} V(\vec{r} ; s) \delta^{3}\left(\vec{r}-\vec{r}^{\prime}\right)+\frac{g}{(2 \pi)^{3}} \iint d \vec{q} K\left(q^{2} ; s\right) V(\vec{r} ; s) e^{-q r} \int d r^{\prime \prime} e^{i q r "} T\left(r^{\prime \prime}, r^{\prime} ; s\right)
$$

Set $T\left(\vec{r}, \vec{r}^{\prime} ; s\right)=\frac{g}{(2 \pi)^{3}} V(\vec{r} ; s) F\left(\vec{r}, \vec{r}^{\prime} ; s\right)$, Eq. (2.3) becomes:

$$
F\left(\vec{r}, \vec{r}^{\prime} ; s\right)=\delta^{3}\left(\vec{r}-\vec{r}^{\prime} ; s\right)+\frac{g}{(2 \pi)^{3}} \int d q K\left(q^{2} ; s\right) e^{-i q r} \times \int d r^{\prime \prime} e^{i q r "} V\left(r^{\prime \prime} ; s\right) F\left(\vec{r}^{\prime \prime}, \vec{r}^{\prime} ; s\right)
$$

The symbol $\hat{L}_{r}=K\left(-\nabla_{r}^{2} ; s\right)$ is the pseudo- differential operator.

After some simple transformations, Eq. (2.3) is rewritten an operator form as:

$$
F\left(\vec{r}, \vec{r}^{\prime} ; s\right)=\delta^{(3)}\left(\vec{r}-\vec{r}^{\prime}\right)+g \hat{L}_{r}\left[V(\vec{r} ; s) F\left(\vec{r}, \vec{r}^{\prime} ; s\right)\right]
$$


Eq. (2.5) is the operator form of the Logunov-Tavkhelizde equation [15].

Within the framework of the quasi-potential approach, the quasi-potential is defined by expanding it into infinite series in order of the interaction constant. This corresponds to the expansion of perturbation amplitudes on the mass shell. The approximate solution of Eq. (2.5) is found in the lowest order of the quasi-potential. Using this method, the relativistic eikonal expression of the scattering amplitude was found in quantum field theory with large energy and small momentum transfer [9].

\section{The Modified Perturbation Theory}

In quantum field theory, scattering problems are mostly solved by peturbation expansion. However, in some cases, in order to solve the problem smoothly, we must improve this method in an expansion that is called the modified pertubation method proposed by Fradkin $^{1}$ [12-14]. Apply it to Eq. (2.6), we can write the solution of this equation in the form.

$$
F\left(\vec{r}, \vec{r}^{\prime} ; s\right)=\frac{1}{(2 \pi)^{3}} \int d \vec{k} \exp [W(\vec{r}, \vec{k} ; s)] e^{-i \vec{k}\left(\vec{r}-\vec{r}^{\prime}\right)}
$$

Substitute Eq. (2.5) into Eq. (3.1), after some simple calculations, we get:

$$
\exp [W(\vec{r}, \vec{k} ; s)]=1+g \hat{L}_{r}\{V(\vec{r} ; s) \exp (W(\vec{r}, \vec{k} ; s)-i \vec{k} \vec{r})\} \exp (i \vec{k} \vec{r})
$$

Here the expression $W(\vec{r}, \vec{k} ; s)$ in the exponent of the RHS of Eq. (3.2) can be expanded in order of the g-coupling constant.

$$
W(\vec{r}, \vec{k} ; s)=\sum_{n=1}^{\infty} g^{n} W_{n}(\vec{r}, \vec{k} ; s)
$$

We transform both the left and right sides of Eq. (3.2) and get:

$$
\begin{aligned}
& 1+\sum_{n=1}^{\infty} g^{n} W_{n}+\frac{1}{2 !}\left(\sum_{n=1}^{\infty} g^{n} W_{n}\right)^{2}+\frac{1}{3 !}\left(\sum_{n=1}^{\infty} g^{n} W^{n}\right)^{3}+\cdots \\
& =1+g\left[\hat{L}_{r}\left\{V(\vec{r} ; s)\left(1+\sum_{n=1}^{\infty} g^{n} W_{n}+\frac{1}{2 !}\left(\sum_{n=1}^{\infty} g^{n} W_{n}\right)^{2}+\frac{1}{3 !}\left(\sum_{n=1}^{\infty} g^{n} W_{n}\right)^{3}+\cdot \cdot\right)\right\}\right. \\
& \left.+V(\vec{r} ; s)\left\{1+\sum_{n=1}^{\infty} g^{n} W_{n}+\frac{1}{2 !}\left(\sum_{n=1}^{\infty} g^{n} W_{n}\right)^{2}+\frac{1}{3 !}\left(\sum_{n=1}^{\infty} g^{n} W^{n}\right)^{3}+\cdots\right\} K\left(\vec{k}^{2} ; s\right)\right] .
\end{aligned}
$$

To compare the terms on both sides of Eq. (3.4) in the same order in the g-coupling constant, we derive the first, second, and third order approximation terms, respectively.

\footnotetext{
${ }^{1}$ The interpretation of the pertubation theory from the view of the diagrammatic technique is as follows. The typical Feynman denominator of the standard pertubation theory is of the form (A): $\left(p+\sum q_{i}\right)^{2}+m^{2}-i \varepsilon=p^{2}+m^{2}+2 p \sum q_{i}+\left(\sum q_{i}\right)^{2}$, where $p$ is the external momentum of the scalar (spinor) particle, and the $q_{i}$ are virtual momenta of radiation quanta. The lowest order approximation (A) of modified theory is equivalent to summing all Feynman diagrams with the replacement $\left(\sum q_{i}\right)^{2}=\sum\left(q_{i}\right)^{2}$ in each denominator (A). The modified pertubation theory thus corresponds to a small correction of the radiation quanta and is often called the $q_{i} q_{j}$ approximation. In the framework of functional integration this approximation is called the straight-line path approximation i.e. high-energy particles move along Feynman paths, which are practically rectilinear.
} 


$$
\begin{gathered}
W_{1}=\hat{L}_{r}(V(\vec{r} ; s))+V(\vec{r} ; s) K\left(\vec{k}^{2} ; s\right)=\int d \vec{q} V(\vec{q} ; s) \exp (-i \vec{q} \vec{r}) K\left((\vec{q}+\vec{k})^{2} ; s\right) \\
\mathrm{W}_{2}=-\frac{W_{1}^{2}}{2 !}+\int d \vec{q}_{1} d \vec{q}_{2} V\left(\vec{q}_{1}\right) V\left(\vec{q}_{2}\right) K\left(\left(\vec{k}+\vec{q}_{1}\right)^{2} ; s\right) K\left(\left(\vec{k}+\vec{q}_{1}+\vec{q}_{2}\right)^{2} ; s\right) \exp \left(-i\left(\vec{q}_{1}+\vec{q}_{2}\right) \vec{r}\right) \\
W_{3}=-\frac{W_{1}^{3}}{3 !}+\int d \vec{q}_{1} d \vec{q}_{2} d \vec{q}_{3} V\left(\vec{q}_{1}\right) V\left(\vec{q}_{2}\right) V(\vec{q} 3) \\
\times K\left(\left(\vec{k}+\vec{q}_{1}+\vec{q}_{2}\right)^{2} ; s\right) \times K\left(\left(\vec{k}+\vec{q}_{1}+\vec{q}_{2}+\vec{q}_{3}\right)^{2} ; s\right) \exp \left(-i\left(\vec{q}_{1}+\vec{q}_{2}+\vec{q}_{3}\right) \vec{r}\right)
\end{gathered}
$$

We are interested in finding the asymptotic scattering amplitude at high energy and t-fixed momentum transfer. We keep the first two approximations (the leading and the first approximation terms):

$$
e^{W(r, p ; s)}=e^{W_{1}(r, \vec{p} ; s)}\left[1+g^{2} W_{2}\left(r, \vec{p}^{\prime} ; s\right)+\ldots\right]
$$

where $W_{1}$ and $W_{2}$ were determined by Eqs. (3.5) and (3.6), respectively.

Take the z-axis along the $\left(\vec{p}+\vec{p}^{\prime}\right)$ momentum of the incident particles and use Mandelstam variables [14], we have:

$$
\begin{aligned}
K\left[\left(\vec{q}+\vec{p}^{\prime}\right)^{2} ; s\right] & =\left.\frac{1}{\sqrt{\left(\vec{q}+\vec{p}^{\prime}\right)^{2}+m^{2}}} \frac{1}{\left(\vec{q}+\vec{p}^{\prime}\right)^{2}-\frac{s}{4}+m^{2}-i \varepsilon}\right|_{s \rightarrow \infty ; t-\text { fixed }} \\
& =\frac{2}{s\left(q_{z}-i \varepsilon\right)}\left[1-\frac{3 q_{z}+q_{\perp}^{2}+\vec{q}_{z} \vec{\Delta}_{\perp}}{\sqrt{s}\left(q_{z}-i \varepsilon\right)}\right]+O\left(\frac{1}{s^{2}}\right)
\end{aligned}
$$

Substitute Eq. (3.9) into Eqs. (3.5) and (3.6), we get:

$$
W_{1}=\left(\frac{W_{10}}{s}\right)+\left(\frac{W_{11}}{s \sqrt{s}}\right)+O\left(\frac{1}{s^{2}}\right), W_{2}=\left(\frac{W_{20}}{s^{2} \sqrt{s}}\right)+O\left(\frac{1}{s^{3}}\right),
$$

where the $W_{10} ; W_{11}$ and $W_{20}$ terms are calculated in detail in Ref. [14]:

$$
\begin{gathered}
W_{10}=2 i \int_{-\infty}^{z} d z^{\prime} \int d q_{z} V\left(q_{z} ; s\right) e^{-i\left|\vec{x}_{\perp}+z^{\prime}\right| q_{2}}=2 i \int_{-\infty}^{z} d z^{\prime} V\left(\sqrt{\vec{x}_{\perp}^{2}+z^{\prime 2}} ; s\right) \\
\mathrm{W}_{11}=-6 V\left(\sqrt{\vec{x}_{\perp}^{2}+z^{\prime 2}} ; s\right)+2\left(-\vec{\nabla}_{\perp}^{2}-i \vec{\Delta}_{\perp} \vec{\nabla}_{\perp}\right) \int_{-\infty}^{z} d z^{\prime}\left(z-z^{\prime}\right) V\left(\sqrt{\vec{x}_{\perp}^{2}+z^{\prime 2}} ; s\right) . \\
W_{20}=-4 i\left\{3 \int_{-\infty}^{z} d z^{\prime} V^{2}\left(\sqrt{\vec{x}_{\perp}^{2}+z^{\prime 2}} ; s\right)+\left[\vec{\nabla}_{\perp} \int_{-\infty}^{z^{\prime}} d z^{\prime \prime} V\left(\sqrt{\vec{x}_{\perp}^{2}+z^{\prime \prime 2}} ; s\right)\right]^{2}\right\} .
\end{gathered}
$$

To determine an asymptotic expression with certain precision, we should only write the leading term and the first approximation term of $\mathrm{W}$ in the following form.

$$
\exp [W(\vec{r}, \vec{k} ; s)]=\exp \left[g W_{1}(\vec{r}, \vec{k} ; s)+g^{2} W_{2}(\vec{r}, \vec{k} ; s)+\cdots\right] .
$$

Replace Eq. (3.14) into Eq. (2.2), after some calculations, we have:

$$
T\left(\vec{p}, \vec{p}^{\prime} ; s\right)=\frac{1}{(2 \pi)^{3}} \int d \vec{r} d \vec{r}^{\prime} d \vec{k} V(\vec{r} ; s) e^{i\left(\vec{p}-\vec{p}^{\prime}\right) \vec{r}} e^{\frac{g}{s} W_{10}} e^{\frac{g}{s \sqrt{s}} W_{11}+\frac{g^{2}}{s^{2} \sqrt{s}} W_{20}+\cdots \cdot} e^{-i \vec{k}\left(\vec{r}-\vec{r}^{\prime}\right)} .
$$


At high energy and fixed momentum transfer, we have the expansion:

$$
e^{\frac{g}{s \sqrt{s}} W_{11}+\frac{g^{2}}{s^{2} \sqrt{s}} W_{20}+\cdots} \approx 1+\frac{g}{s \sqrt{s}} W_{11}+\frac{g^{2}}{s^{2} \sqrt{s}} W_{20}+\ldots
$$

Then integrate with respect to radial direction (from inward to outward scattering zone) that is perpendicular to the z-direction. Here in the eikonal approximation we consider the trajectory of scattered particles close to the classical trajectory, along with the notation: $\vec{\Delta}_{\perp}=\vec{p}-\vec{p}^{\prime} ; t=-\left(\vec{p}-\vec{p}^{\prime}\right)^{2}$.

Therefore, Eq. (3.15) can be written in the form:

$$
\left.T(s ; t)\right|_{\substack{s \rightarrow \infty \\ t-f x \operatorname{xid}}}=\frac{g}{(2 \pi)^{3}} \int d \vec{x}_{\perp}^{2} d z e^{i \vec{\Lambda}_{\perp} \vec{r}_{\perp}} V\left(\sqrt{\vec{x}_{\perp}^{2}+z^{2}} ; s\right) e^{\left(\frac{g}{s} W_{10}\right)}\left(1+\frac{g}{s \sqrt{s}} W_{11}+\frac{g^{2}}{s^{2} \sqrt{s}} W_{20}+\cdots\right) .
$$

The first term in Eq. (3.18) describes the leading eikonal behavior of the scattering amplitude ${ }^{2}$

$$
\left.T(s, t)\right|_{s \rightarrow \infty ; t-\text { fixed }} ^{\text {leading }}=\frac{g}{2 i(2 \pi)^{3}} \int d \vec{x}_{\perp}^{2} d z e^{i \vec{\Delta}_{\perp} \vec{x}_{\perp}}\left\{\exp \left[\frac{2 i g}{s} \int_{-\infty}^{z} d z V\left(\sqrt{\vec{x}_{\perp}^{2}+z^{2}} ; s\right)\right]-1\right\}
$$

and the remaining terms determine the corrections. These correction terms have relative magnitude propotional to $1 / \sqrt{s}$, smaller than the leading term. The dependence on the s-energy of the leading term and the correction terms is also found by means of the functional integration [14].

\section{Asymptotic Terms of the Scattering Amplitude}

To further illustrate the results obtained in Section 3, in this section we consider the interaction of the two nucleons by exchanging the scalar particle (with zero spin such as $\pi^{ \pm} ; \pi^{0} ; K^{ \pm} ; K^{0} \cdots$ ) in the case of the Yukawa and the Gaussian potentials. These smooth potentials appear naturally when describing the scattering process with being explicitly accounted for the contribution of particles in the intermediate states [14]. However, due to the complexity of integrals, we only compute the numerical result only for the leading term and the first correction term of the scattering amplitude.

The interaction of charged hadron particles is often described by two types of potentials such as the Coulomb potential for electromagnetic interaction and nuclear potential for interactions in short regions (the strong interaction). The choice of nuclear local potentials here must follow reasons:

(1) They must be smooth function and have singularity.

(2) For the basic properties of hadron scattering at large and small angles.

(3) In high energy and the fixed momentum transfer, the total scattering cross-section will decrease according to the exponential rule when square of the momentum is increased to match the emprical data [4].

\subsection{Cross-Section with the Yukawa Potential}

The interaction potential of two nucleons through the exchange of scalar particles is called the Yukawa potential [16]. It has form:

$$
V(\vec{r} ; s)=V_{0} \frac{e^{-\mu|\vec{r}|}}{|\vec{r}|}=V_{0} \frac{e^{-\mu \sqrt{\bar{x}_{\perp}^{2}+z^{2}}}}{\sqrt{\vec{x}_{\perp}^{2}+z^{2}}}
$$

\footnotetext{
${ }^{2}$ This is well-known Mollier-Glauber representation.
} 
where $V_{0}$ is the magnitude coupling constant, $\mu$ is another scaling constant which is related to $\mathrm{R}-$ the effective size where the potential is nonrezo as $\mu=(1 / R)$.

Substitute Eq. (4.1) into Eq. (3.23) and note that the collision parameters are small compared to the $\lambda$-wavelength of the scattering particle [18]. $\left|x_{\perp}\right| \leq \lambda=\frac{1}{p_{0}} ; p_{0}=(\sqrt{\mathrm{s}} / 2) \rightarrow \infty$ and $\left|x_{\perp}\right| \rightarrow 0$, performing the necessary calculations according to appendix A, we obtain the scattering amplitude.

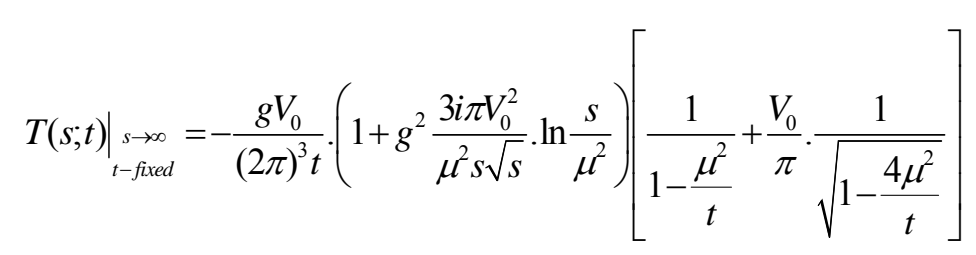

Here, we considered the scattering at high energy and based on the experimental results [17] in proton scattering in hydrogen atoms, we take the following constants: $V_{0}=1$ (atomic unit of energy $\left(\mathrm{m}_{\mathrm{e}} \alpha \mathrm{c}^{2}=27.2 \mathrm{eV}\right.$ ); $\mu=2 a_{0}\left(a_{0}\right.$ is the Bohr radius, in the atomic unit of length), the coupling constant $\mathrm{g}=1 / 137$, and $\Delta_{\perp}^{2}=-t=4 q^{2} \sin ^{2} \theta / 2 \quad\left(\theta\right.$ is the scattering angle), so that $\frac{\mu^{2}}{t}=\frac{\mu^{2}}{4 q^{2} \sin ^{2}(\theta / 2)}$, we also retain the term that depends on $t$. We obtain the differential cross-section.

$$
\frac{d \sigma}{d \Omega}=|T(s ; t)|^{2}=\frac{g^{2}}{(2 \pi)^{6} 16 q^{4} \sin ^{4}(\theta / 2)} \cdot\left[\frac{1}{1-\frac{\mu^{2}}{4 q^{2}} \cdot \frac{1}{\sin ^{2}(\theta / 2)}}+\frac{1}{\pi} \cdot \frac{1}{\sqrt{1-\frac{\mu^{2}}{q^{2}} \cdot \frac{1}{\sin ^{2}(\theta / 2)}}}\right]^{2}
$$

Next, we plot the dependence of the differential cross section on the scattering angle and the momentum of the incoming particle in the center system.

Fig. 1 shows that at small angles, the diferential cross section of the proton scattering in the hydrogen atom increases as it has only strong interaction, but with the scattering of two protons it decreases due to the electromagnetic interaction is not considered.

From Fig. 2, the scattering is negligible at high momentum and large angles (dashed lines) and the differential cross section only increase at small angles (solid lines).

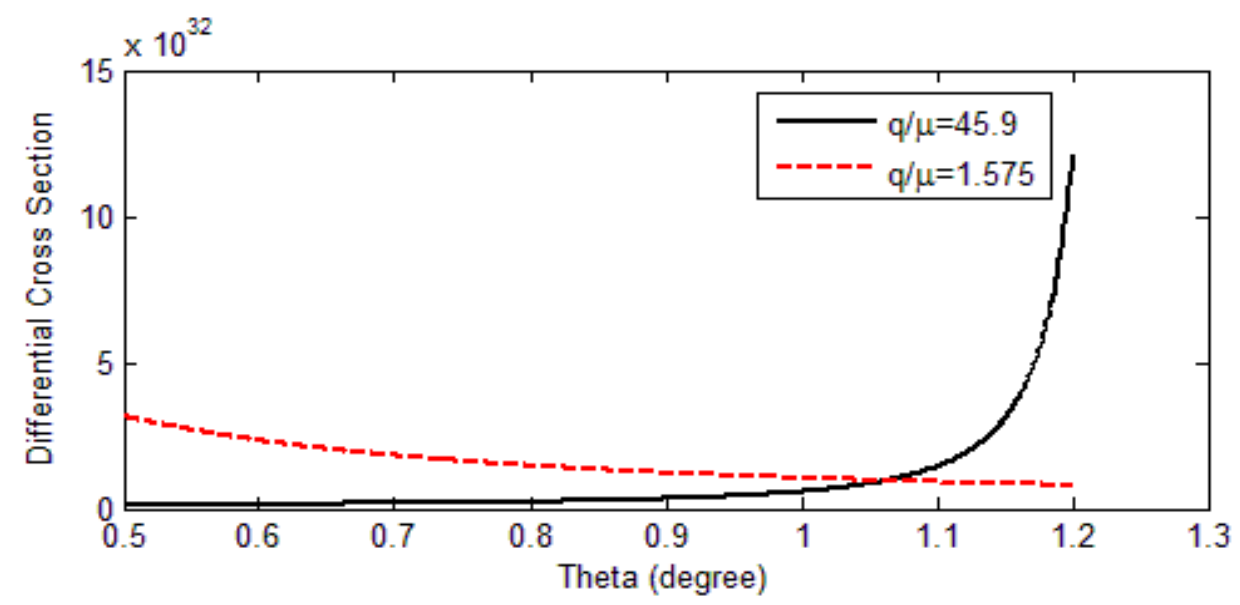

Fig. 1 The dependence of differential cross section on small scattering angle.

a. The solid line is the scattering of protons in hydrogen atoms.

b. The dashed line is the scattering of two protons at energy $50 \mathrm{MeV}$. 


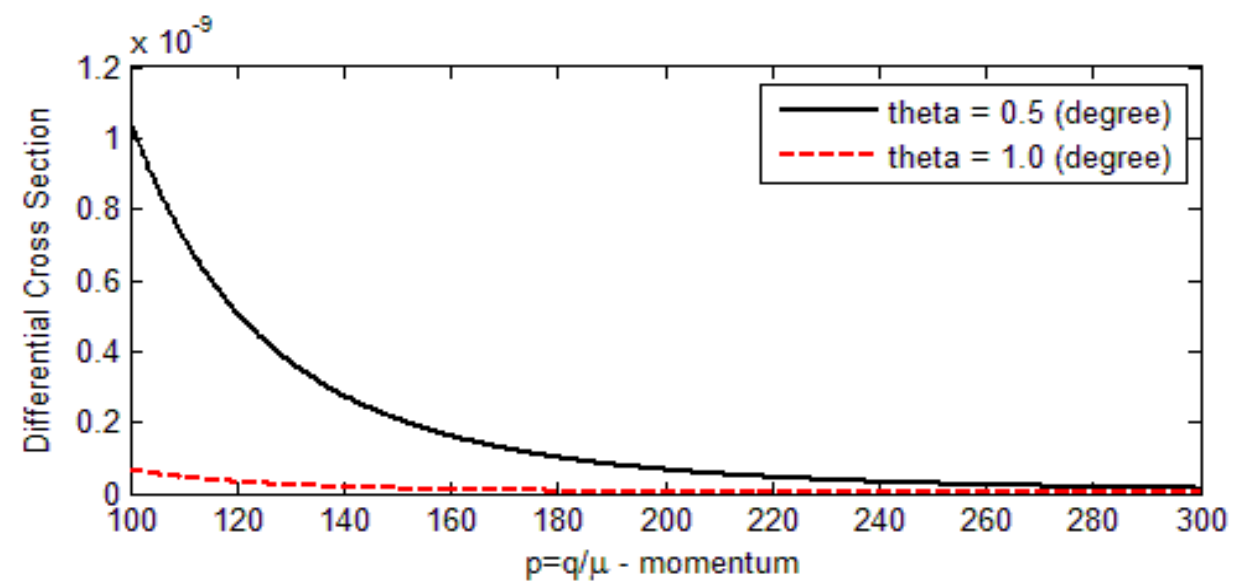

Fig. 2 The dependence of the differential cross section on the momentum of the incoming particle at small angles.

\subsection{Cross-Section with the Gaussian Potential}

Now, we consider the Gaussian potential of following form [17]:

$$
V(r)=V_{0} e^{-\alpha r^{2}}=V_{0} \exp \left(-\frac{r^{2}}{2 R^{2}}\right)
$$

where $V_{0}$ is the magnitude scaling constant, $R$ is the effective size where the potential is non-zero, and $\alpha$ is another scaling constant, $\alpha=\left(1 / 2 R^{2}\right)$.

For this case, we perform calculations similar to the case of the Yukawa potential. In particular, we have (see appendix B):

$$
\left.T(s ; t)\right|_{\substack{s \rightarrow \infty \\ t-\text { fixed }}} \approx\left[-\frac{3 V_{0} g}{32 \pi^{2}} \frac{1}{\alpha \sqrt{\alpha}}-i \frac{3\left(V_{0} g\right)^{2} \sqrt{\pi}}{32 \pi^{2} \alpha^{2} s}\right] \frac{1}{2 \sin (\theta / 2)}
$$

The differential cross-section is:

$$
\frac{d \sigma}{d \Omega}=|T(s ; t)|^{2}=\left[\left(\frac{3 V_{0} g}{32 \pi^{2}} \frac{1}{\alpha \sqrt{\alpha}}\right)^{2}+\left(\frac{3\left(V_{0} g\right)^{2} \sqrt{\pi}}{32 \pi^{2} \alpha^{2} s}\right)^{2}\right] \frac{1}{4 \sin ^{2}(\theta / 2)}=\left(\frac{3 V_{0} g}{32 \pi^{2}}\right)^{2} \frac{1}{\alpha^{3}}\left[1+\frac{V_{0}^{2} g^{2} \pi}{\alpha s}\right] \frac{1}{4 \sin ^{2}(\theta / 2)}
$$

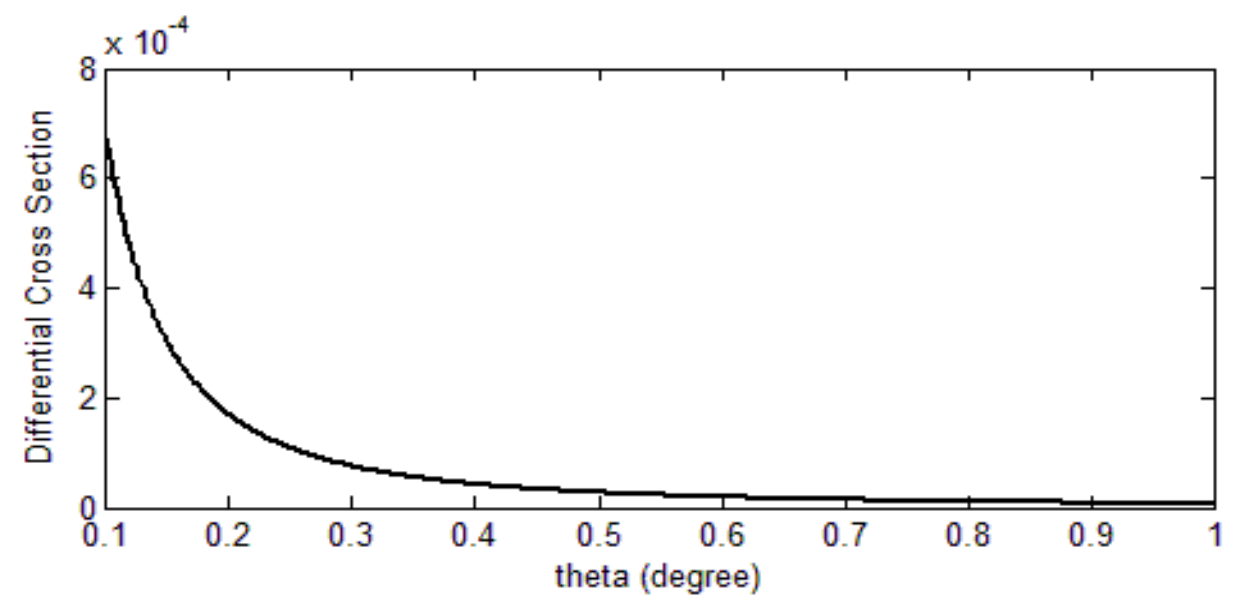

Fig. 3 The dependence of differential cross-section on small scattering angles. 
Here, we considered the scattering at high energy, $S \rightarrow \infty$, and based on experimental results [17] in the scattering of alpha particles with energy $166 \mathrm{MeV}$ in center of mass reference frame withV0 $=-41.6 \mathrm{MeV}$, the coupling constant $\mathrm{g}=1 / 137, \sqrt{\pi \alpha}=7.1$.

Fig 3., we plot the dependence of the differential cross section on the scattering angle in the center system. This result shows that with small scattering angles, the differential cross section is significant at small scattering angles.

\section{Conclusion}

Within the framework of the quasi-potential approach, the modified perturbation has been studied and developed. Applying it to the problem of interaction of two scalar particles in quantum field theory demonstrates that the well known Moliere-Glauber representation for the case of the Logunov-Tavkhelidze equation is suitable at high energy and fixed momentum transfer. The diagram for calculating the leading term of the scattering amplitude and the first and second order terms is also found. Using the Yukawa and Gaussian potentials, the scatering amplitudes and the differential cross sections have been obtained, respectively. Numerical results will be advantageous for comparison with experimental results.

The modified pertubation theory can be used for study the gravitational interaction of two particles that we expect to study in the near future [18, 19$]$.

We would like to thank Profs. Nguyen Ai Viet, Dang Van Soa for helpful discussions. This work was completed with support from the National Foundation for Science and Technology of Vietnam (NAFOSTED)
2017.

\section{References}

[1] Bethe, H. A., and Salpeter, E. E. 1951. "Quantum Mechanics of One- and Two-Electron Atoms." Phys. Rev. 82: 309 .

[2] Itzykson, C., and Zuber, J.-B. 2006. Quantum Field Theory. Dover: McGraw Hill.

[3] Kusaka, K., and Williams, A. G. 1995. Phys. Rev. D51 7026.

[4] Garvesevanishvili, V. R., Matveev, V. A., Slepchenko, L. A., and Tavkhelidze, A. N. 1971. "Quasipotential Theory of High Energy Hadron Scattering." Physical Review D Particles \& Fields 4 (3): 849-61.

[5] Kadyshevsky, V. A., Mir-Kashimov, R. M., and Skachkov, N. B. 1968. "Quasi-Potential Approach and the Expansion in Relativistic Spherical Functions." Nuovo Cimento 55A: 233.

[6] Todorov, I. T. 1971. "Quasipotential Approach to the Two-Body Problems in Quantum Field Theory." Phys. Rev. D 3 (10): 2352.

[7] Filippov, A.T. 1970.Fiz. Elm. Chast. At. Yadra 10: 501

[8] Logunov, A. A., and Tavkhelidze, A. N. 1969. "Problems of Theoretical Physics.” Dedicaled to N. N. Bogoliubov on the Occasion of his $60^{\text {th }}$ Birthday (Nauka 1969).

[9] Garvesevanishvili, V. R., Matveev, V. A., Slepchenko, L. A., and Tavkhelidze, A. N. 1969. Phys. Lett. 29B: 191.

[10] Arbuzov, A. B. 1994. Nuovo Cimento 107A (8): 1263.

[11] Nguyen, V. H., and Faustov, R. N. 1964. Nucl. Phys. 53: 337.

[12] Fradkin, E. S., Esposito, V., and Termini, S. 1970. Revista del Nuovo Cimento 2 (1): 498-560.

[13] Kuleshov, S. P., Matveev, V. A., Savelyev, M. V., Sissakian, A. N., and Smondyev, M. A. 1971. TMF 6: 36.

[14] Han, N. S., Yen, L. H., and Xuan, N. N. 2012. IJMPA 27 (1): 1250004.

[15] Logunov, A. A., and Tavkhelidze, A. N. 1963. Nuovo Cimento 29: 380.

[16] Efimov, G.V. 1970. TMF 179: 695.

[17] Wallace, S. J. 1973. Annals of Physics 78: 190.

[18] Han, N. S. 2000. EPJC16: 547.

[19] Han, N. S., and Xuan, N. N. 2002. EPJC 24: 640 


\section{Appendix A: Some calculations for amplitude scattering in the Yukawa potential}

Using some integrals in Ref. [14], we have,

$$
I=\int_{-\infty}^{\infty} d z V(\vec{r} ; s)=\frac{V_{0}}{2 \pi} K_{0}\left(\mu\left|x_{\perp}\right|\right)
$$

Following Eqs.(3.11)-(3.13), the leading term has the form.

$$
\mathrm{W}_{1} \approx \frac{W_{10}}{s}=\left.\frac{2 i}{s} \int_{-\infty}^{z} d z^{\prime} V\left(\sqrt{\vec{x}_{\perp}^{2}+z^{\prime 2}} ; s\right)\right|_{z \rightarrow \infty}=\left(\frac{2 i}{s}\right) \times\left[\frac{V_{0}}{2 \pi} K_{0}\left(\mu\left|x_{\perp}\right|\right)\right]=\frac{i V_{0}}{\pi s} K_{0}\left(\mu\left|x_{\perp}\right|\right)
$$

and the first correction term is:

$$
\mathrm{W}_{2} \approx \frac{W_{20}}{s^{2} \sqrt{s}}=-\left.\frac{4 i}{s^{2} \sqrt{s}}\left\{3 \int_{-\infty}^{z} d z^{\prime} V^{2}\left(\sqrt{\vec{x}_{\perp}^{2}+z^{\prime 2}} ; s\right)+\left[\vec{\nabla}_{\perp} \int_{-\infty}^{z^{\prime}} d z^{\prime \prime} V\left(\sqrt{\vec{x}_{\perp}^{2}+z^{\prime \prime 2}} ; s\right)\right]^{2}\right\}\right|_{z \rightarrow \infty}
$$

With $K_{1}\left(\mu\left|x_{\perp}\right|\right)$ being the first order MacDonald function, $K_{1}\left(\mu\left|x_{\perp}\right|\right)=-\frac{1}{\mu} \frac{d}{d x_{\perp}}\left(K_{0}\left(\mu\left|x_{\perp}\right|\right)\right)$.

Consider the integral,

$$
\int_{0}^{\infty} \frac{e^{-2 \mu r}}{r^{2}} d z=-\frac{\pi}{\mu\left|x_{\perp}\right|} K_{1}\left(2 \mu\left|x_{\perp}\right|\right)
$$

The first term in parenthesis of Eq. (A.3) is,

$$
3 \int_{-\infty}^{\infty} d z V^{2}\left(\sqrt{\vec{x}_{\perp}^{2}+z^{2}} ; s\right)=3 V_{0}^{2} \int_{0}^{\infty} d z \frac{e^{-2 \mu r}}{r^{2}}=3 V_{0}^{2} \times\left[-\frac{\pi}{\mu\left|x_{\perp}\right|} K_{1}\left(2 \mu\left|x_{\perp}\right|\right)\right]=-\frac{3 \pi V_{0}^{2}}{\mu\left|x_{\perp}\right|} K_{1}\left(2 \mu\left|x_{\perp}\right|\right)
$$

The second term in parenthesis of Eq. (A.3) is,

$$
\left[\vec{\nabla}_{\perp} \int_{-\infty}^{\infty} d z V\left(\sqrt{\vec{x}_{\perp}^{2}+z^{2}} ; s\right)\right]^{2}=\left[\frac{d I}{d x_{\perp}}\right]^{2}=\left(\frac{V_{0}}{2 \pi}\right)^{2}\left[\frac{d}{d x_{\perp}} K_{0}\left(\mu\left|x_{\perp}\right|\right)\right]^{2}=\frac{\mu^{2} V_{0}^{2}}{\pi^{2}}\left[K_{1}\left(\mu\left|x_{\perp}\right|\right)\right]^{2}
$$

thus

$$
\mathrm{W}_{2} \approx-\frac{4 i V_{0}^{2}}{s^{2} \sqrt{s}}\left\{-\frac{3 \pi}{\mu\left|x_{\perp}\right|} K_{1}\left(2 \mu\left|x_{\perp}\right|\right)+\frac{\mu^{2}}{\pi^{2}}\left[K_{1}\left(\mu\left|x_{\perp}\right|\right)\right]^{2}\right\}
$$

So that,

$$
\frac{\mathrm{W}_{2}}{\mathrm{~W}_{1}}=\frac{4 \pi V_{0}}{s \sqrt{s}} \times \frac{\left\{\frac{3}{\mu\left|x_{\perp}\right|} K_{1}\left(2 \mu\left|x_{\perp}\right|\right)-\frac{\mu^{2}}{\pi^{3}}\left[K_{1}\left(\mu\left|x_{\perp}\right|\right)\right]^{2}\right\}}{K_{0}\left(\mu\left|x_{\perp}\right|\right)}
$$

If the collision parameters are small compared to the wavelength of the scattering particle $\left|x_{\perp}\right| \leq \lambda=\frac{1}{p_{0}} ; p_{0}=(\sqrt{s} / 2) \rightarrow \infty$. If $\lambda=\frac{1}{p_{0}}$ and $\left|x_{\perp}\right| \rightarrow 0$ then we use the approximation, 


$$
\left.K_{0}\left(\mu\left|x_{\perp}\right|\right)\right|_{\left|x_{\perp}\right| \rightarrow 0} \approx \ln ^{2} \frac{s}{\mu^{2}} ;\left.K_{1}\left(\mu\left|x_{\perp}\right|\right)\right|_{\left|x_{\perp}\right| \rightarrow 0} \approx \frac{\sqrt{s}}{\mu} \ln \frac{s}{\mu^{2}} ;\left.K_{1}\left(2 \mu\left|x_{\perp}\right|\right)\right|_{\left|x_{\perp}\right| \rightarrow 0} \approx \frac{\sqrt{s}}{2 \mu} \ln \frac{s}{4 \mu^{2}}
$$

Therefore,

$$
\begin{gathered}
\frac{\mathrm{W}_{2}}{\mathrm{~W}_{1}}=\frac{4 \pi^{2} V_{0}}{\sqrt{s}} \times \frac{\left(\frac{3}{4 \mu^{2}} \ln \frac{s}{4 \mu^{2}}-\frac{1}{\pi^{3}} \ln ^{2} \frac{s}{\mu^{2}}\right)}{\ln ^{2} \frac{s}{\mu^{2}}}=\frac{3 \pi^{2} V_{0}}{\mu^{2} \sqrt{s}} \times \frac{\ln \frac{s}{4 \mu^{2}}}{\ln ^{2} \frac{s}{\mu^{2}}}-\frac{4 V_{0}}{\pi \sqrt{s}} \\
\text { At high energy } s \rightarrow \infty \text { then } \frac{\mathrm{W}_{2}}{\mathrm{~W}_{1}}=\frac{3 \pi^{2} V_{0}}{\mu^{2} \sqrt{s}} \times \frac{1}{\ln \frac{s}{\mu^{2}}}
\end{gathered}
$$

The scattering amplitude is determined by using the energy terms $\mathrm{W}_{1}, \mathrm{~W}_{2}$.

$$
\begin{aligned}
\left.T(s ; t)\right|_{\substack{s \rightarrow \infty \\
t-\text { fixed }}} & =\frac{g}{(2 \pi)^{3}} \int d \vec{x}_{\perp}^{2} d z e^{i \vec{\Delta}_{\perp} \vec{\perp}_{\perp}} V\left(\sqrt{\vec{x}_{\perp}^{2}+z^{2}} ; s\right) e^{\left(g W_{1}\right)}\left(1+g^{2} W_{2}\right) \\
& =\frac{g}{(2 \pi)^{3}} \int d \vec{x}_{\perp}^{2} d z e^{i \vec{\Delta}_{\perp} \vec{r}_{\perp}} V\left(\sqrt{\vec{x}_{\perp}^{2}+z^{2}} ; s\right) \exp \left(2 i \int_{-\infty}^{z} d z^{\prime} V\left(\sqrt{\vec{x}_{\perp}^{2}+z^{\prime 2}} ; s\right)\right)\left(1+g^{2} W_{2}\right)
\end{aligned}
$$

As above calculation, we have $\int_{-\infty}^{z} d z^{\prime} V\left(\sqrt{\vec{x}_{\perp}^{2}+z^{\prime 2}} ; s\right)=\frac{V_{0}}{2 \pi} K_{0}\left(\mu\left|x_{\perp}\right|\right)$.

Thus,

$$
\begin{gathered}
\left.T(s ; t)\right|_{\substack{s \rightarrow \infty \\
t-f \text { ixed }}}=\frac{g}{(2 \pi)^{3}} \int d \vec{x}_{\perp}^{2} e^{i \vec{\Delta}_{\perp} \vec{x}_{\perp}} \frac{V_{0}}{2 \pi} K_{0}\left(\mu\left|x_{\perp}\right|\right) \exp \left(\frac{i V_{0}}{\pi} K_{0}\left(\mu\left|x_{\perp}\right|\right)\right)\left(1+g^{2} W_{2}\right) \\
\approx\left(1+g^{2} \frac{3 i \pi V_{0}^{2}}{\mu^{2} s \sqrt{s}} \cdot \ln \frac{s}{\mu^{2}}\right)\left[\frac{g V_{0}}{(2 \pi)^{4}} \int d \vec{x}_{\perp}^{2} e^{i \vec{\Delta}_{\perp} \vec{x}_{\perp}} K_{0}\left(\mu\left|x_{\perp}\right|\right)+\frac{i g V_{0}^{2}}{\pi(2 \pi)^{4}} \int d \vec{x}_{\perp}^{2} e^{i \vec{i}_{\perp} \vec{x}_{\perp}} K_{0}^{2}\left(\mu\left|x_{\perp}\right|\right)\right] \\
=\left(1+g^{2} \frac{3 i \pi V_{0}^{2}}{\mu^{2} s \sqrt{s}} \cdot \ln \frac{s}{\mu^{2}}\right)\left[\frac{g V_{0}}{(2 \pi)^{3}} \cdot \frac{1}{\mu^{2}-t}+\frac{g V_{0}^{2}}{(2 \pi)^{4}} \cdot \frac{1}{t \sqrt{1-\frac{4 \mu^{2}}{t}}} \ln \frac{1-\sqrt{1-\frac{4 \mu^{2}}{t}}}{1+\sqrt{1-\frac{4 \mu^{2}}{t}}}\right]
\end{gathered}
$$

Expanding around $\mu$ small, we obtain:

$$
\left.\left.T(s ; t)\right|_{\substack{t \rightarrow \text { fixed } \\(2 \pi)^{3} t}}=-\frac{g V_{0}}{\mu^{2} s \sqrt{s}} \cdot \ln \frac{s}{\mu^{2}}\right)\left[\frac{1}{1-\frac{\mu^{2}}{t}}+\frac{V_{0}}{\pi} \cdot \frac{1}{\sqrt{1-\frac{4 \mu^{2}}{t}}}\right]
$$




\section{Appendix B: Some calculations for amplitude scattering in the Gaussian potential}

The leading term has the form:

$$
\mathrm{W}_{1} \approx \frac{W_{10}}{s}=\left.\frac{2 i}{s} \int_{-\infty}^{z} d z^{\prime} V\left(\sqrt{\vec{x}_{\perp}^{2}+z^{\prime 2}} ; s\right)\right|_{z \rightarrow \infty}=\left(\frac{2 i}{s}\right) \times V_{0} \int_{-\infty}^{\infty} d z e^{-\alpha r^{2}}=\frac{2 i V_{0}}{s} \sqrt{\frac{\pi}{\alpha}} e^{-\alpha x_{\perp}^{2}}
$$

and the first correction term is:

$$
\mathrm{W}_{2} \approx \frac{W_{20}}{s^{2} \sqrt{s}}=-\frac{4 i}{s^{2} \sqrt{s}}\left\{3 V_{0}^{2} \int_{-\infty}^{\infty} d z e^{-2 \alpha r^{2}}+\left[V_{0} \vec{\nabla}_{\perp} \int_{-\infty}^{\infty} d z e^{-\alpha r^{2}}\right]^{2}\right\}=-\frac{4 i V_{0}^{2}}{s^{2} \sqrt{s}}\left\{3 \sqrt{\frac{\pi}{2 \alpha}}+4 \pi \alpha\left|x_{\perp}\right|^{2}\right\} e^{-2 \alpha x_{\perp}^{2}}
$$

So that,

$$
\frac{\mathrm{W}_{2}}{\mathrm{~W}_{1}}=-\frac{2 V_{0}}{s \sqrt{s}} \times\left\{\frac{3}{\sqrt{2}}+4 \alpha \sqrt{\pi \alpha}\left|x_{\perp}\right|^{2}\right\} e^{-\alpha x_{\perp}^{2}}
$$

If the collision parameters are small compared to the wavelength of the scattering particle $\left|x_{\perp}\right| \leq \lambda=\frac{1}{p_{0}} ; p_{0}=(\sqrt{s} / 2) \rightarrow \infty$ then we can use the approximation $\left|x_{\perp}\right|=\frac{2}{\sqrt{s}}$.

Therefore,

$$
\frac{\mathrm{W}_{2}}{\mathrm{~W}_{1}} \approx-\frac{2 V_{0}}{s \sqrt{s}} \times\left\{\frac{3}{\sqrt{2}}+\frac{8 \alpha \sqrt{\pi \alpha}}{s}\right\}\left(1-\frac{2 \alpha}{s}\right)=-\frac{3 \sqrt{2} V_{0}}{s \sqrt{s}}+\frac{6 \sqrt{2} \alpha V_{0}}{s^{2} \sqrt{s}}-\frac{16 \alpha \sqrt{\pi \alpha} V_{0}}{s^{2} \sqrt{s}}+\frac{32 \alpha^{2} \sqrt{\pi \alpha} V_{0}}{s^{3} \sqrt{s}}
$$

From Eq. (B.4) pointed out that all terms are reduced respect to the s-energy.

Using Eq. (B.1) for finding the first correction term,

$$
\mathrm{W}_{2} \approx-\frac{4 i V_{0}^{2}}{s^{2} \sqrt{s}}\left\{3 \sqrt{\frac{\pi}{2 \alpha}}+4 \pi \alpha\left|x_{\perp}\right|^{2}\right\} e^{-2 \alpha x_{\perp}^{2}}
$$

And the scattering amplitude with $\mathrm{W}_{1}, \mathrm{~W}_{2}$ terms,

$$
\begin{aligned}
& \left.T(s ; t)\right|_{\substack{s \rightarrow \infty \text { fixed } \\
(2 \pi)^{3}}} \approx \frac{V_{0} g}{\alpha} \int x_{\perp} d \vec{x}_{\perp} J_{0}\left(\vec{\Delta}_{\perp} \vec{x}_{\perp}\right) e^{-\alpha x_{\perp}^{2}}\left(1+\frac{2 i g V_{0}}{s} \sqrt{\frac{\pi}{\alpha}} e^{-\alpha x_{\perp}^{2}}\right)\left[1-\frac{4 i g^{2} V_{0}^{2}}{s^{2} \sqrt{s}}\left(3 \sqrt{\frac{\pi}{2 \alpha}}+4 \pi \alpha\left|x_{\perp}\right|^{2}\right) e^{-2 \alpha x_{\perp}^{2}}\right] \\
& =C_{1} \int x_{\perp} d \vec{x}_{\perp} J_{0}\left(\vec{\Delta}_{\perp} \vec{x}_{\perp}\right) e^{-\alpha x_{\perp}^{2}}+C_{2} \int x_{\perp} d \vec{x}_{\perp} J_{0}\left(\vec{\Delta}_{\perp} \vec{x}_{\perp}\right) e^{-2 \alpha x_{\perp}^{2}}+C_{31} \int x_{\perp} d \vec{x}_{\perp} J_{0}\left(\vec{\Delta}_{\perp} \vec{x}_{\perp}\right) e^{-3 \alpha x_{\perp}^{2}} \\
& +C_{32} \int x_{\perp}^{3} d \vec{x}_{\perp} J_{0}\left(\vec{\Delta}_{\perp} \vec{x}_{\perp}\right) e^{-3 \alpha x_{\perp}^{2}}+C_{41} \int x_{\perp} d \vec{x}_{\perp} J_{0}\left(\vec{\Delta}_{\perp} \vec{x}_{\perp}\right) e^{-4 \alpha x_{\perp}^{2}}+C_{42} \int x_{\perp}^{3} d \vec{x}_{\perp} J_{0}\left(\vec{\Delta}_{\perp} \vec{x}_{\perp}\right) e^{-4 \alpha x_{\perp}^{2}}
\end{aligned}
$$

where

$$
C_{1}=\frac{V_{0} g}{(2 \pi)^{3}} \sqrt{\frac{\pi}{\alpha}} ; C_{2}=i \frac{\left(V_{0} g\right)^{2}}{(2 \pi)^{2} \alpha s} ; C_{31}=-\frac{3 i\left(V_{0} g\right)^{3}}{2 \sqrt{2} \alpha \pi^{2} s^{2} \sqrt{s}} ; C_{32}=-\frac{2 i\left(V_{0} g\right)^{3} \sqrt{\pi \alpha}}{\pi^{2} s^{2} \sqrt{s}} ; C_{41}=\frac{3\left(V_{0} g\right)^{4}}{\sqrt{2} \pi^{2} \alpha s^{3} \sqrt{s}} \sqrt{\frac{\pi}{\alpha}} ; C_{42}=\frac{4\left(V_{0} g\right)^{4}}{\pi s^{3} \sqrt{s}} .(\mathrm{B} .7)
$$

Using the formula,

$$
\int_{0}^{\infty} x^{\mu} e^{-\alpha x^{2}} J_{v}(\beta x) d x=\frac{\Gamma\left(\frac{1}{2} \mu+\frac{1}{2} v+1\right)}{\beta \alpha^{\frac{1}{2} \mu} \Gamma(v+1)} \exp \left(-\frac{\beta^{2}}{8 \alpha}\right) M_{\frac{1}{2} \mu, \frac{1}{2} v}\left(-\frac{\beta^{2}}{4 \alpha}\right)
$$


Then the final expression of the scattering amplitude is

$$
\begin{aligned}
\left.T(s ; t)\right|_{\substack{s \rightarrow \infty \\
t-f \text { ixed }}} & C_{1} \cdot \frac{3 \sqrt{\pi}}{2\left|\vec{\Delta}_{\perp}\right| \sqrt{\alpha}} \exp \left(\frac{t}{8 \alpha}\right) M_{\frac{1}{2}, 0}\left(\frac{t}{4 \alpha}\right)+C_{2} \cdot \frac{3 \sqrt{\pi}}{2\left|\vec{\Delta}_{\perp}\right| \sqrt{2 \alpha}} \exp \left(\frac{t}{16 \alpha}\right) M_{\frac{1}{2}, 0}\left(\frac{t}{8 \alpha}\right) \\
& +C_{31} \frac{3 \sqrt{\pi}}{2\left|\vec{\Delta}_{\perp}\right| \sqrt{3 \alpha}} \exp \left(\frac{t}{24 \alpha}\right) M_{\frac{1}{2}, 0}\left(\frac{t}{12 \alpha}\right)+C_{32} \cdot \frac{15 \sqrt{\pi}}{4\left|\vec{\Delta}_{\perp}\right| \sqrt{3 \alpha}} \exp \left(\frac{t}{24 \alpha}\right) M_{\frac{3}{2}, 0}\left(\frac{t}{12 \alpha}\right) \\
& +C_{41} \frac{3 \sqrt{\pi}}{4\left|\vec{\Delta}_{\perp}\right| \sqrt{\alpha}} \exp \left(\frac{t}{32 \alpha}\right) M_{\frac{1}{2}, 0}\left(-\frac{t}{16 \alpha}\right)+C_{42} \cdot \frac{15 \sqrt{\pi}}{2\left|\vec{\Delta}_{\perp}\right| \sqrt{\alpha}} \exp \left(-\frac{t}{32 \alpha}\right) M_{\frac{3}{2}, 0}\left(\frac{t}{16 \alpha}\right)
\end{aligned}
$$

Note that,

$$
M_{\frac{1}{2}, 0}\left(\frac{t}{4 \alpha}\right)=\exp \left(-\frac{t}{8 \alpha}\right) \cdot \sqrt{\frac{t}{4 \alpha}} ; M_{\frac{3}{2}, 0}\left(\frac{t}{4 \alpha}\right)=\exp \left(-\frac{t}{8 \alpha}\right) \cdot \sqrt{\frac{t}{4 \alpha}}\left(1-\frac{t}{4 \alpha}\right)
$$

Thus,

$$
\begin{aligned}
\left.T(s ; t)\right|_{\substack{s \rightarrow \infty \\
t \rightarrow \text { fixed }}} & =C_{1} \cdot \frac{3 \sqrt{\pi}}{2\left|\vec{\Delta}_{\perp}\right| \sqrt{\alpha}} \cdot \sqrt{\frac{t}{4 \alpha}}+C_{2} \cdot \frac{3 \sqrt{\pi}}{2\left|\vec{\Delta}_{\perp}\right| \sqrt{2 \alpha}} \sqrt{\frac{t}{8 \alpha}}+C_{31} \frac{3 \sqrt{\pi}}{2\left|\vec{\Delta}_{\perp}\right| \sqrt{3 \alpha}} \sqrt{\frac{t}{12 \alpha}} \\
& +C_{32} \cdot \frac{15 \sqrt{\pi}}{4\left|\vec{\Delta}_{\perp}\right| \sqrt{3 \alpha}} \sqrt{\frac{t}{12 \alpha}}\left(1-\frac{t}{12 \alpha}\right)+C_{41} \frac{3 \sqrt{\pi}}{4\left|\vec{\Delta}_{\perp}\right| \sqrt{\alpha}} \sqrt{\frac{t}{16 \alpha}}+C_{42} \cdot \frac{15 \sqrt{\pi}}{2\left|\vec{\Delta}_{\perp}\right| \sqrt{\alpha}} \sqrt{\frac{t}{16 \alpha}}\left(1-\frac{t}{16 \alpha}\right)
\end{aligned}
$$

Substitute $\left|\Delta_{\perp}\right|=2 q \sin (\theta / 2)$ into Eq. (B.9), we have:

$$
\left.T(s ; t)\right|_{\substack{s \rightarrow \infty \\ t-\text { fixed }}}=\left[-C_{1} \cdot \frac{3 \sqrt{\pi}}{4 \alpha}-C_{2} \cdot \frac{3 \sqrt{\pi}}{8 \alpha}-C_{31} \frac{3 \sqrt{\pi}}{12 \alpha}-C_{32} \cdot \frac{15 \sqrt{\pi}}{24 \alpha}\left(1-\frac{t}{12 \alpha}\right)-C_{41} \frac{3 \sqrt{\pi}}{16 \alpha}-C_{42} \cdot \frac{15 \sqrt{\pi}}{8 \alpha}\left(1-\frac{t}{16 \alpha}\right)\right] \frac{\sqrt{t}}{2 q \sin (\theta / 2)}(\mathrm{B} .10)
$$

At high energy, $S \rightarrow \infty$, retain the lower order terms in proportion to the $1 / \mathrm{s}$, we have:

$$
\left.T(s ; t)\right|_{\substack{t \rightarrow \text { fixed } \\ \text { (n) }}} \approx\left[-C_{1} \cdot \frac{3 \sqrt{\pi}}{4 \alpha}-C_{2} \cdot \frac{3 \sqrt{\pi}}{8 \alpha}\right] \frac{1}{2 \sin (\theta / 2)} \approx\left[-\frac{3 \lambda g}{32 \pi^{2}} \frac{1}{\alpha \sqrt{\alpha}}-i \frac{3(\lambda g)^{2} \sqrt{\pi}}{32 \pi^{2} \alpha^{2} s}\right] \frac{1}{2 \sin (\theta / 2)}
$$

